\title{
FULLY AUTOMATIC IMAGE-BASED REGISTRATION OF UNORGANIZED TLS DATA
}

\author{
Martin Weinmann, Boris Jutzi \\ Institute of Photogrammetry and Remote Sensing, Karlsruhe Institute of Technology (KIT) \\ Kaiserstr. 12, 76128 Karlsruhe, Germany \\ \{martin.weinmann, boris.jutzi\}@kit.edu
}

KEY WORDS: Laser Scanning, TLS, point cloud, registration, image-based, graph-based, automation.

ABSTRACT:

The estimation of the transformation parameters between different point clouds is still a crucial task as it is usually followed by scene reconstruction, object detection or object recognition. Therefore, the estimates should be as accurate as possible. Recent developments show that it is feasible to utilize both the measured range information and the reflectance information sampled as image, as 2D imagery provides additional information. In this paper, an image-based registration approach for TLS data is presented which consists of two major steps. In the first step, the order of the scans is calculated by checking the similarity of the respective reflectance images via the total number of SIFT correspondences between them. Subsequently, in the second step, for each SIFT correspondence the respective SIFT features are filtered with respect to their reliability concerning the range information and projected to $3 \mathrm{D}$ space. Combining the $3 \mathrm{D}$ points with $2 \mathrm{D}$ observations on a virtual plane yields 3D-to-2D correspondences from which the coarse transformation parameters can be estimated via a RANSAC-based registration scheme including the EPnP algorithm. After this coarse registration, the 3D points are again checked for consistency by using constraints based on the 3D distance, and, finally, the remaining 3D points are used for an ICP-based fine registration. Thus, the proposed methodology provides a fast, reliable, accurate and fully automatic image-based approach for the registration of unorganized point clouds without the need of a priori information about the order of the scans, the presence of regular surfaces or human interaction.

\section{INTRODUCTION}

The automatic registration of point clouds acquired with a terrestrial laser scanner (TLS) is still of great interest. Each point cloud represents dense and accurate 3D information about surfaces of objects in the local area around the scanner with respect to a local coordinate frame. However, usually multiple scans from different locations have to be recorded to obtain a full scene coverage. Hence, a registration process has to be carried out which transforms all point clouds into a common coordinate frame.

Standard approaches for calculating the transformation parameters between two partially overlapping point clouds are based on the Iterative Closest Point (ICP) algorithm (Besl \& McKay, 1992) and different variants of it (Rusinkiewicz \& Levoy, 2001). The ICP algorithm minimizes the difference between two point clouds. For large numbers of points, however, the ICP algorithm shows a high computational effort which is due to the iterative processing scheme. Hence, it seems quite feasible to extract relevant information from the point clouds which can be used for registration. Such relevant information may for example be derived via the distribution of the points within each point cloud by using the normal distributions transform (NDT) either on 2D scan slices (Brenner et al., 2008) or in 3D (Magnusson et al., 2007).

Urban environments or scenes containing industrial installations usually contain regular surfaces of which various types of geometric features might arise. Simple features which are likely to occur and useful for registration are lines (Stamos \& Leordeanu, 2003) derived from the range information sampled as range images. Other commonly used features which are extracted directly from the point clouds are planes (Dold \& Brenner, 2004; Brenner et al., 2008; Pathak et al. 2010a; Pathak et al. 2010b) or more complex geometric features like spheres, cylinders or tori (Rabbani et al., 2007). However, all these feature types representing geometric primitives are not suited in scenes without regular surfaces. In addition to regular surfaces, scans in urban scenes might also contain a typical skyline. This border between the sky and a set of buildings shows special features when using a cylindrical projection model for sampling the range information to panoramic range images covering $360^{\circ}$ in the horizontal direction, e.g. extrema or flat regions which are suited for a coarse alignment of two scans (Nüchter et al., 2011). As such features strongly depend on the scene content, this approach is not suited if the skyline is less distinctive and thus not sufficient for registration purposes.

In the presence of cluttered scenes, descriptors representing local surface patches are more appropriate. Such descriptors may be derived from geometric curvature or normal vectors of the local surface (Bae \& Lichti, 2004; Bae \& Lichti 2008). Further approaches which are suitable for more complex scenes are based on extracting special feature points in the range images in order to support the registration process (Barnea \& Filin, 2008; Steder et al., 2010).

Currently, most of the terrestrial laser scanners can not only measure the distance to 3D scene points but also capture either co-registered camera images or panoramic reflectance images representing the respective energy of the backscattered laser light. Therefore, several approaches are based on the use of 2D imagery as the images provide additional information about the local area around the scanner which might not always be represented in the range measurements. Hence, the registration of two point clouds can be supported by using reliable feature correspondences between the respective camera or reflectance images. For this purpose, different kinds of features can be used, but most of the current image-based approaches are based on the use of feature points or keypoints. Many of the imagebased approaches use SIFT features to detect distinctive 2D feature points by which point correspondences between two images can be detected. These features can be extracted from the co-registered camera images (Bendels et al., 2004; AlManasir \& Fraser, 2006; Barnea \& Filin, 2007) or from the reflectance images (Böhm \& Becker, 2007; Wang \& Brenner 2008; Kang et al., 2009). For all point correspondences, the respective feature points are projected into $3 \mathrm{D}$ space and thus lead to a much smaller set of 3D points used for registration.

In this paper, a method for a fully automatic registration of a large number of unorganized scans is proposed. Reaching a 
high level of automation also including the sorting of the scans is essential as most of the current approaches are based on pairwise registration for which an already known order of the scans is assumed. For pairwise registration, a modification of a fast and automatic image-based registration approach which has been published recently (Weinmann et al., 2011) and which is also suited for scenes without regular surfaces is presented. In contrast to this approach, only those correspondences with reliable range information are used and, instead of a refinement step, an ICP-based fine registration is introduced. Thus, besides being very fast, the proposed algorithm does neither depend on a priori information about the order of the scans nor on the presence of regular surfaces.

The paper is organized as follows. In Section 2, the processing chain of the proposed algorithm is outlined. As it will be shown, the algorithm can be divided into the two major steps of organizing the TLS data and carrying out a successive pairwise registration which are presented in Section 3 and Section 4 in detail. In Section 5, the performance of the proposed algorithm is proved by processing 11 point clouds of a benchmark TLS data set. The capability of the proposed method is discussed in Section 6 with respect to accuracy, reliability and performance. Finally, conclusions and suggestions for future work are outlined in Section 7.

\section{METHODOLOGY}

The registration approach proposed in this paper aims at reaching a high level of automation and simultaneously getting fast, reliable and accurate results. As illustrated in Figure 1, the approach can be divided into two major parts. The first part deals with the organization of unorganized point clouds which is later required for a successive pairwise registration. Hence after the acquisition of TLS data, special features have to be extracted which are suitable for organizing the scans. The second part focuses on the registration of the point clouds. Using reliable $3 \mathrm{D}$ points derived via the previously extracted features and introducing their projections onto a virtual plane yields 3D-to-2D correspondences. These are used for receiving a coarse registration of the point clouds which is followed by a fine registration in order to improve the accuracy of the results. The scheme of this briefly summarized methodology is presented in detail in Section 3 and Section 4.

\section{ORGANIZATION OF TLS DATA}

The current registration approaches address a fully automatic registration of different scans of a scene. To further increase the level of automation, the proposed algorithm first organizes the scans automatically, which yields a structure for successive pairwise registration. This is done by considering the recorded scans (Section 3.1), extracting distinctive features and feature correspondences between the scans (Section 3.2) and using a graph-based approach (Section 3.3).

\subsection{Data Set and Reference Values}

In the following, the quality of the proposed registration approach is demonstrated with a benchmark TLS data set provided by the University of Hanover. This set consists of 12 upright scans and 8 tilted scans which were acquired in the German city of Hanover in an area called Holzmarkt, and the respective reference values for the relative orientation between the scans. The scans were recorded with a Riegl LMS-Z360i scanner and contain information about the $3 \mathrm{D}$ coordinates of object points as well as the corresponding reflectance information. Covering $360^{\circ}$ in the horizontal direction and $90^{\circ}$ in the vertical direction with a single shot measurement accuracy of $12 \mathrm{~mm}$ and an angular resolution of $0.12^{\circ}$ up to a range of approximately $200 \mathrm{~m}$, each scan returns 2.25 million $3 \mathrm{D}$ points from a regular scan area of $3000 \times 750$ points being represented as panoramic reflectance image (Wang \& Brenner, 2008). The reflectance and range information derived from the scan at scan position 01 are visualized in Figure 2.

In order to check the quality of the automatically calculated registration results, accurate reference values are needed. The provided reference values are based on the use of artificial targets and a manual alignment which yields an expected accuracy of the scan positions in the low millimeter range. For testing the proposed algorithm, a subset consisting of 11 upright scans with a spacing of approximately $5 \mathrm{~m}$ is used.

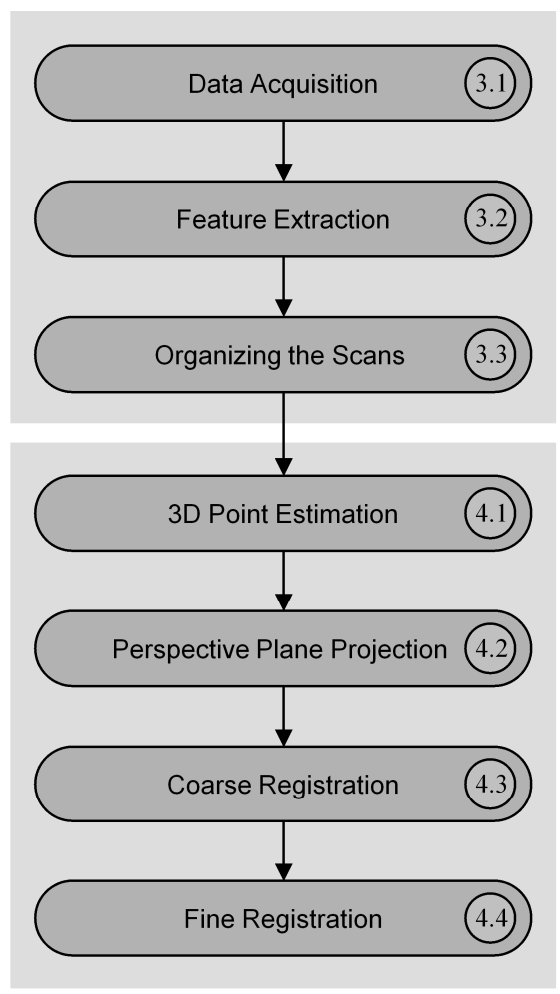

Figure 1. Processing chain of the proposed approach.

\subsection{Feature Extraction}

Once several scans have been acquired, the next step consists of extracting distinctive features. Here, the Scale Invariant Feature Transform (SIFT) (Lowe, 2004) is utilized for detecting such distinctive keypoints in an image derived from the TLS data and extracting local feature descriptors which are invariant to image scaling and image rotation, and robust with respect to image noise, changes in illumination and small changes in viewpoint. These descriptors allow for locating correspondences between different images and, finally, to derive common image objects. As the descriptors are represented as vectors, they can be compared by considering Euclidean distances. An effective measure describing the distinctiveness of a keypoint can be derived from the ratio of the Euclidean distances of a descriptor belonging to a keypoint in one image to the nearest neighbor and the second nearest neighbor in the other image. This ratio has to be below a given threshold $t_{\mathrm{des}}$, which can vary between 0 and 1. For practical purposes and different applications, distinctive features arise when using a threshold between $t_{\mathrm{des}}=$ 0.6 and $t_{\mathrm{des}}=0.8$. As the feature correspondences used for 
registration should be reliable, a threshold of $t_{\mathrm{des}}=0.66$ is used. This means that the distance of a descriptor belonging to a SIFT feature in image $i$ to the nearest neighbor in image $j$ is only about $2 / 3$ of the distance to the second nearest neighbor.

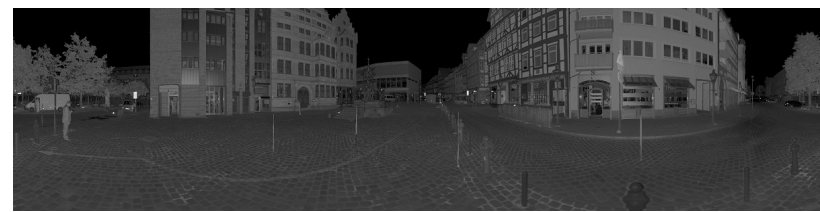

(a)

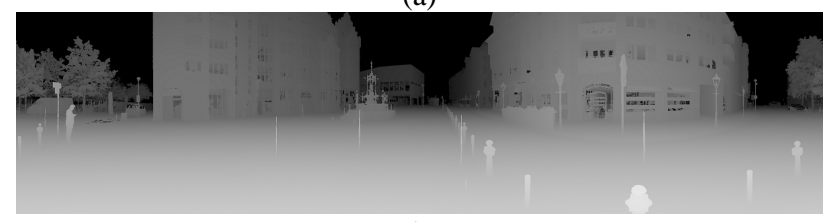

(b)

Figure 2. Visualization of the captured TLS data: (a) reflectance and (b) range information.

In order to check the similarity of the scans, the number of SIFT features between image pairs from all available positions is calculated and stored in the confusion matrix C. For this purpose, the same scan IDs are used as in the provided data set. The diagonal elements $\mathbf{C}(i, i)$ represent the total number of SIFT features extracted in the respective reflectance image $i$. As can be seen in Table 1, the confusion matrix is not necessarily symmetric which depends on the calculated ratio of the Euclidean distances of a feature descriptor to the nearest and second nearest neighbor. If, for a feature descriptor derived from image $i$, the nearest neighbor and the second nearest neighbor in image $j$ are a little more distinctive as required, this ratio is below the threshold $t_{\mathrm{des}}$ and thus meets the constraint. In the reverse case, when comparing a feature descriptor derived from image $j$ to feature descriptors derived from image $i$, it might occur that the nearest neighbor and the second nearest neighbor are more similar which causes a ratio above the threshold $t_{\text {des }}$.

\subsection{Organizing Large Numbers of Scans by Similarity}

When dealing with a large number of scans, it might be desirable to reach a high level of automation. This will also include automatically sorting the scans for pairwise registration so that the error between estimated and real position is minimal. Therefore, a graph-based algorithm is proposed here.

Any set of unorganized point clouds can directly be represented as a graph, where the nodes represent the scans and the edges are weighted with the total number of SIFT correspondences between the respective scans. In the most general case, every node is connected with every other node which results in a complete graph. As mentioned before, the confusion matrix $\mathbf{C}$ is not necessarily symmetric and therefore, a directed graph is used instead of an undirected graph. Hence, the entry $\mathbf{C}(i, j)$ of the confusion matrix represents the weight of an unidirectional edge from node $i$ to node $j$.

The first step towards organizing the point clouds consists of an initialization which can be done via selecting a defined initial scan. Alternatively, it would be possible to use other criterions if only the relations between the scans are of importance, e.g. the node from which the edge with the maximum weight within the graph starts. This initial set containing exactly one node is then iteratively expanded until it contains all nodes of the graph. Each iteration starts with searching unidirectional edges from the actual set of nodes to the remaining nodes and the edge with the maximum weight leads to the node by which the actual set is expanded. Resulting from the selected connections, a structure can be generated which represents the order of the scans for the automatic pairwise registration. For the given confusion matrix C (Table 1), the resulting structure for a successive pairwise registration process is shown in Figure 3.

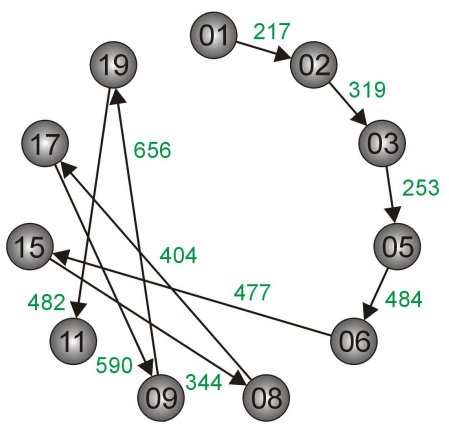

Figure 3. Resulting scheme for successive pairwise registration: The scans are labeled with their ID and the connections used for further calculations are labeled with the number of detected SIFT correspondences between the respective reflectance images.

\section{REGISTRATION OF TLS DATA}

After calculating the order of the scans by checking the similarity of the respective reflectance images, a pairwise registration of successive scans can be carried out. For this purpose, the already calculated 2D SIFT features leading to

\begin{tabular}{|c|c|c|c|c|c|c|c|c|c|c|c|}
\hline Scan ID & $\mathbf{0 1}$ & $\mathbf{0 2}$ & $\mathbf{0 3}$ & $\mathbf{0 5}$ & $\mathbf{0 6}$ & $\mathbf{0 8}$ & $\mathbf{0 9}$ & $\mathbf{1 1}$ & $\mathbf{1 5}$ & $\mathbf{1 7}$ & $\mathbf{1 9}$ \\
\hline $\mathbf{0 1}$ & 4986 & 217 & 63 & 45 & 33 & 58 & 41 & 28 & 44 & 62 & 39 \\
\hline $\mathbf{0 2}$ & 229 & 5663 & 319 & 100 & 59 & 80 & 43 & 48 & 35 & 46 & 38 \\
\hline $\mathbf{0 3}$ & 88 & 308 & 5967 & 253 & 120 & 56 & 47 & 68 & 57 & 38 & 56 \\
\hline $\mathbf{0 5}$ & 70 & 114 & 277 & 6200 & 484 & 78 & 58 & 68 & 131 & 68 & 84 \\
\hline $\mathbf{0 6}$ & 31 & 70 & 124 & 466 & 6682 & 169 & 68 & 56 & 477 & 134 & 71 \\
\hline $\mathbf{0 8}$ & 86 & 96 & 53 & 78 & 163 & 6867 & 205 & 64 & 328 & 404 & 99 \\
\hline $\mathbf{0 9}$ & 39 & 34 & 37 & 56 & 68 & 158 & 5571 & 330 & 78 & 577 & 656 \\
\hline $\mathbf{1 1}$ & 17 & 24 & 37 & 40 & 44 & 41 & 277 & 4061 & 24 & 134 & 408 \\
\hline $\mathbf{1 5}$ & 61 & 40 & 59 & 129 & 503 & 344 & 82 & 30 & 7154 & 211 & 53 \\
\hline $\mathbf{1 7}$ & 53 & 56 & 34 & 60 & 121 & 379 & 590 & 169 & 240 & 6159 & 361 \\
\hline $\mathbf{1 9}$ & 21 & 25 & 43 & 51 & 54 & 84 & 629 & 482 & 42 & 344 & 4852 \\
\hline
\end{tabular}

Table 1. Number of SIFT correspondences between the reflectance images of different scans within the chosen subset. The values can be summarized in the confusion matrix $\mathbf{C}$ and the entry $\mathbf{C}(i, j)$ of this matrix denotes the number of point correspondences found when all descriptors derived from image $i$ are compared to the nearest neighbor and the second nearest neighbor derived from image $j$. 
correspondences have to be projected to $3 \mathrm{D}$ space, and the reliability of the calculated $3 \mathrm{D}$ points has to be checked with respect to range information (Section 4.1) before registration. The following registration is based on $2 \mathrm{D}$ projections of the reliable 3D points onto a virtual plane (Section 4.2), and divided into a coarse registration (Section 4.3) and a fine registration (Section 4.4).

\subsection{D Point Estimation}

As SIFT features are determined with subpixel accuracy, the respective $3 \mathrm{D}$ information has to be interpolated as the measured values are only available on the regular scan raster. A reliable 3D point corresponding to a SIFT feature can however only be generated, if all of the four nearest points on the scan raster contain valid range information. The measured points which arise from objects in the scene will probably provide a smooth surface whereas points corresponding to the sky or points along edges of the objects might be very noisy. Therefore, points have to be discarded if they do not lie on the surface of any object in the scene.

The provided scans are already filtered with respect to minimum values of the backscattered energy (Figure 4a). Additionally, the proposed algorithm considers the standard deviation $\sigma$ of the values within a $3 \times 3$ neighborhood of each pixel in the range image in order to avoid unreliable range information at edges of scene objects. If the standard deviation $\sigma$ of the respective range values is larger than a predefined threshold $t_{\text {std }}$ which is selected to $t_{\mathrm{std}}=0.1 \mathrm{~m}$, the range information of the center pixel is not reliable, otherwise the range information of the center pixel is assumed to be reliable (Figure $4 \mathrm{~b}$ ). Combining these constraints yields a $2 \mathrm{D}$ confidence map $\mathbf{M}_{C}$ which is illustrated in Figure $4 \mathrm{c}$.

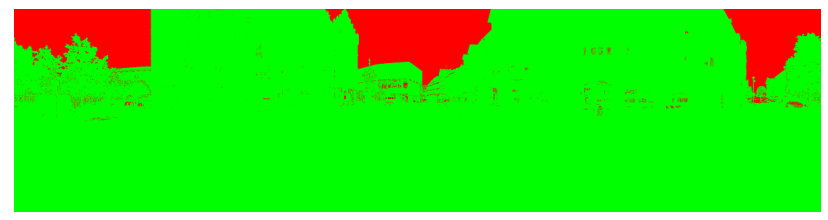

(a)

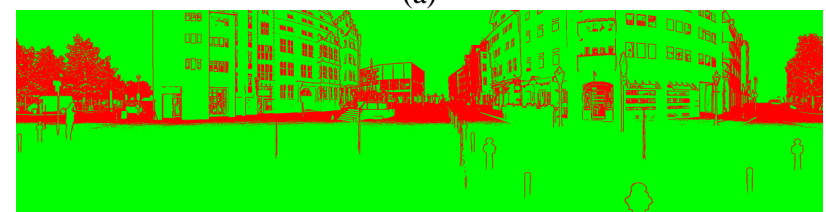

(b)

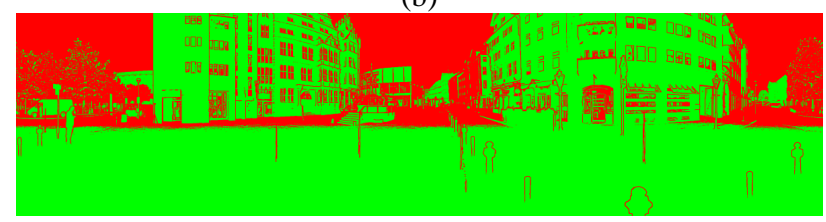

(c)

Figure 4. Confidence map for the scan at scan position 01: (a) information filtered with respect to reflectance, (b) information filtered with respect to the standard deviation $\sigma$ using a threshold value of $t_{\text {std }}=0.1 \mathrm{~m}$ and (c) the resulting confidence map $\mathbf{M}_{C}$. The reliable points are shown in green, the unreliable ones in red.

\subsection{Perspective Plane Projection}

The reflectance images have been sampled using a spherical projection. For the registration, however, it is useful to get the coordinates of the extracted and reliable $3 \mathrm{D}$ points $\mathbf{X}_{i}$ projected onto a 2D image plane of a virtual camera in order to use powerful algorithms of computer vision applications. The respective transformation can be described via

$$
\mathbf{x}_{i}=\mathbf{K}[\mathbf{R} \mid \mathbf{t}] \mathbf{X}_{i}
$$

where the matrix $\mathbf{K}$ is the calibration matrix of a virtual camera, and the matrix $\mathbf{R}$ and the vector $\mathbf{t}$ describe the rotation and the translation of this virtual camera with respect to the local coordinate frame of the laser scanner. In the registration process, $\mathbf{R}$ refers to the local coordinate frame so that the virtual camera has the same orientation as the laser scanner and looks into the horizontal direction (Weinmann et al., 2011). Besides, the position of the virtual camera is assumed to equal the location of the laser scanner and therefore, the translation vector is set to $\mathbf{t}=\mathbf{0}$. As a consequence of introducing a virtual camera plane, any parameters can be used for the focal lengths of the camera in $x$ - and $y$-direction as well as for the coordinates of the principal point. Furthermore, the image plane has not necessarily to be limited on a finite area and all points behind the camera may also be included by mapping them onto the virtual plane via symmetric constraints as they represent the $2 \mathrm{D}$ projections onto the virtual plane of a second camera looking in the opposite direction. Thus, instead of creating synthetic camera images and using these for registration (Forkuo \& King, 2004), only a few points are projected with subpixel accuracy.

\subsection{Coarse Registration using EPnP and RANSAC}

Once 3D-to-2D correspondences are known, the problem of pose estimation is the same as when using a camera instead of a laser scanner. Recently, the Efficient Perspective-n-Point (EPnP) algorithm has been proposed as a non-iterative method to estimate the exterior orientation or pose of a camera from a set of $n$ correspondences between 3D points $\mathbf{X}_{i}$ of a scene and their $2 \mathrm{D}$ projections $\mathbf{x}_{i}$ onto the image plane (Moreno-Noguer et al., 2007; Lepetit et al., 2009). The EPnP algorithm is based on the idea of expressing the $n$ known $3 \mathrm{D}$ scene points $\mathbf{X}_{i}$ as a weighted sum of four virtual and non-coplanar control points $\mathbf{C}_{j}$ for general configurations. The weights $\alpha_{i j}$ remain unchanged when transferring this relation to camera coordinates and therefore, the points $\mathbf{X}_{i}^{c}$ can be expressed via the control points $\mathbf{C}_{j}^{c}$ which leads to

$$
w_{i}=\left[\begin{array}{c}
\mathbf{x}_{i} \\
1
\end{array}\right]=\mathbf{K} \mathbf{X}_{i}^{c}=\mathbf{K} \sum_{j=1}^{4} \alpha_{i j} \mathbf{C}_{j}^{c}
$$

for $i=1, \ldots, n$, where $\mathbf{K}$ describes the camera matrix. The scalar projective parameters $w_{i}$ can be substituted by

$$
w_{i}=\sum_{j=1}^{4} \alpha_{i j} Z_{j}^{c}
$$

via the $Z^{c}$ coordinates of the control points. Concatenating the resulting equations for all $\mathrm{n} 3 \mathrm{D}$-to-2D correspondences yields a linear system $\mathbf{M x}=\mathbf{0}$ with $\mathbf{x}=\left[\mathbf{C}_{1}^{c T}, \mathbf{C}_{2}^{c T}, \mathbf{C}_{3}^{c T}, \mathbf{C}_{4}^{c T}\right]^{T}$ and a $2 n \times 12$ matrix $\mathbf{M}$. The solution $\mathbf{x}$ then leads to the camera coordinates $\mathbf{X}_{i}^{c}$. Once the world coordinates and the camera coordinates of the $3 \mathrm{D}$ points are known, the rotation and translation parameters aligning both coordinate systems can be retrieved via standard methods (Horn et al., 1988). As the EPnP algorithm considers all 3D-to-2D correspondences without checking their reliability, the quality of the registration results 
can be increased by introducing further constraints. The RANSAC algorithm (Fischler \& Bolles, 1981) provides a good possibility for eliminating outliers and thus reaching a more robust pose estimation. This combination of $\mathrm{EPnP}$ and RANSAC is based on randomly selecting small, but not minimal subsets of seven correspondences for estimating the model parameters, and checking the whole set of correspondences for consistent sample points (Moreno-Noguer et al., 2007; Lepetit et al., 2009).

\subsection{Fine Registration using Outlier Removal and ICP}

The results from the previous coarse registration provide a good a priori alignment which is required for using the ICP algorithm in order to get a fine registration. However, the RANSAC algorithm only considers the quality of the 3D-to-2D correspondences. Hence, the quality of the 3D points has to be considered separately. This is done by eliminating those 3D-to$3 \mathrm{D}$ correspondences for which at least one 3D point arises from periodic shapes of façades and thus from ambiguities in the scene via geometric constraints (Weinmann et al., 2011). The resulting points used for fine registration are very reliable as they have been checked with respect to the reliability of their range information, the quality of the $3 \mathrm{D}$-to-2D correspondences and the quality of the 3D-to-3D correspondences. Therefore, the ICP algorithm is expected to yield very accurate results.

\section{EVALUATION}

The first part of the presented approach can easily be verified if the nodes belonging to the scans are projected onto the reference positions of the respective scans in the scene, which is done in Figure 5.

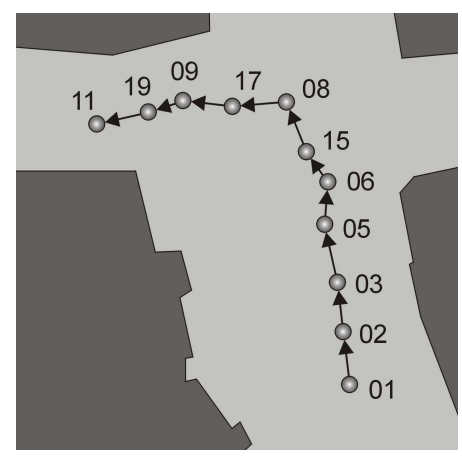

Figure 5. Scans sorted with respect to their reference position in the nadir view of the scene: The streets are colored in bright gray, buildings in dark gray.

Once the scans are sorted, a successive pairwise registration can be carried out. Between the reflectance images of the scans 01 and 02 , a total number of 217 SIFT correspondences has been detected (Table 1) of which 89 are reliable with respect to the range information of the corresponding SIFT features. The respective 3D points of those reliable SIFT correspondences are projected to $3 \mathrm{D}$ space using bilinear interpolation. If for one scan, the absolute transformation parameters with respect to the world coordinate frame are known which is assumed for scan position 01 , the $3 \mathrm{D}$ world coordinates of the calculated $3 \mathrm{D}$ points can easily be determined. For a new scan, the corresponding 2D features with reliable range information are also projected to $3 \mathrm{D}$ space and backprojected onto a virtual plane assigned to the local coordinate frame which yields $2 \mathrm{D}$ observations. Establishing 3D-to-2D correspondences from the $3 \mathrm{D}$ information derived from the first scan and the $2 \mathrm{D}$ observations derived from the new scan allows for using the
EPnP algorithm which has been extended by the RANSAC algorithm for an increased robustness. Subsequently, a consistency check with respect to 3D distances between the 3D points transformed into a common coordinate frame via the coarse estimate of the transformation parameters is carried out. After this geometric outlier removal, the remaining 3D-to-3D correspondences ( 29 between the scans 01 and 02) are used for an ICP-based fine registration. As shown in Figure 6, the absolute position errors after coarse registration are in the range between $12 \mathrm{~mm}$ and $49 \mathrm{~mm}$, and the fine registration yields accurate results with absolute position errors between $9 \mathrm{~mm}$ and $32 \mathrm{~mm}$.

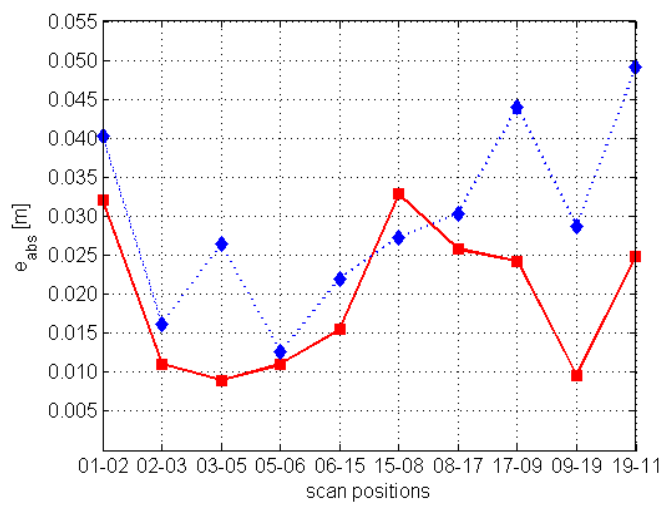

Figure 6. Absolute error between reference and estimated positions for coarse (dotted line with diamonds) and fine registration (solid line with squares).

\section{DISCUSSION}

The presented registration approach was tested in Matlab on a standard PC with $2.83 \mathrm{GHz}$. Although the code is not fully optimized with respect to a possible parallelization on multiple cores and thus only one core is used, the average time required for pairwise registration is about $13 \mathrm{~s}$. Of this time, about $5 \mathrm{~s}$ are needed for calculating the SIFT correspondences, about $7 \mathrm{~s}$ for coarse estimation using a RANSAC-based scheme including EPnP and only $1 \mathrm{~s}$ for consistency checks and ICP on two relatively small subsets each consisting of approximately 100 points. If the ICP algorithm is used for larger subsets, the computational effort increases highly.

Concerning accuracy and performance, the proposed approach is comparable to other image-based approaches (Wang \& Brenner, 2008; Weinmann et al., 2011). As the approach focuses on using only reliable information concerning range, 3D-to-2D correspondences and 3D-to-3D correspondences, the estimated transformation parameters are very reliable which can be seen when comparing them to the reference values (Figure 6). The approach is suited for both urban environments and scenes containing vegetation and does neither depend on regular surfaces nor human interaction. However, one constraint concerning the scene arises as point-like features have to be extracted. Hence, the scene has to be well-structured which is assumed in all image-based approaches using SIFT features.

As the total number of SIFT correspondences decreases with an increasing distance between the respective scan positions which can be seen when considering the entries in the confusion matrix (Table 1) and the reference positions (Figure 5), the presented approach as well as other image-based approaches will not lead to optimal results for larger distances between the scans. For this purpose, approaches based on geometric primitives (Brenner et al., 2008; Rabbani et al., 2007) might be 
more robust in direct comparison, but they assume that regular surfaces can be found in the scene and thus less general scenes.

\section{CONCLUSION AND OUTLOOK}

In this paper, a fully automatic registration approach is presented which is based on both the range information and the reflectance information of terrestrial laser scans. Automatically sorting any number of unorganized scans by means of their similarity and then carrying out a successive fast and accurate pairwise registration, the approach provides a powerful framework suited for typical environments. The approach has been successfully applied to a benchmark TLS data set containing millions of points and been discussed concerning accuracy, reliability and performance. For future work, the approach could be extended by introducing a final global registration over all scans or at least considering those parts of the confusion matrix arising from the similarity of a new scan to all of the already registered scans. This might improve the quality of the estimated transformation parameters and yield an even further increased robustness.

\section{ACKNOWLEDGEMENT}

The authors would like to thank Dr. Claus Brenner and the Institute of Cartography and Geoinformatics at the University of Hanover for providing the TLS data. The data is available at http://www.ikg.uni-hannover.de/index.php?id=413\&L=de and has been accessed in March 2011.

\section{REFERENCES}

Al-Manasir, K., Fraser, C. S., 2006. Registration of terrestrial laser scanner data using imagery. The Photogrammetric Record 21 (115), pp. 255-268.

Bae, K.-H., Lichti, D. D., 2004. Automated registration of unorganised point clouds from terrestrial laser scanners. International Archives of Photogrammetry, Remote Sensing and Spatial Information Sciences 35 (Part B5), pp. 222-227.

Bae, K.-H., Lichti, D. D., 2008. A method for automated registration of unorganised point clouds. ISPRS Journal of Photogrammetry and Remote Sensing 63 (1), pp. 36-54.

Barnea, S., Filin, S., 2007. Registration of terrestrial laser scans via image based features. International Archives of Photogrammetry, Remote Sensing and Spatial Information Sciences 36 (Part 3), pp. 32-37.

Barnea, S., Filin, S., 2008. Keypoint based autonomous registration of terrestrial laser point-clouds. ISPRS Journal of Photogrammetry and Remote Sensing 63 (1), pp. 19-35.

Bendels, G. H., Degener, P., Körtgen, M., Klein, R., 2004. Imagebased registration of 3D-range data using feature surface elements. In: Chrysanthou, Y., Cain, K., Silberman, N., Niccolucci, F. (Eds.), The 5th International Symposium on Virtual Reality, Archaeology and Cultural Heritage, pp. 115-124.

Besl, P. J., McKay, N. D., 1992. A method for registration of 3-D shapes. IEEE Transactions on Pattern Analysis and Machine Intelligence 14 (2), pp. 239-256.

Böhm, J., Becker, S., 2007. Automatic marker-free registration of terrestrial laser scans using reflectance features. In: Gruen, A., Kahmen, H. (Eds.), Optical 3-D Measurement Techniques VIII, pp. 338-344.

Brenner, C., Dold, C., Ripperda, N., 2008. Coarse orientation of terrestrial laser scans in urban environments. ISPRS Journal of Photogrammetry and Remote Sensing 63 (1), pp. 4-18.

Dold, C., Brenner, C., 2004. Automatic matching of terrestrial scan data as a basis for the generation of detailed 3D city models.
International Archives of the Photogrammetry, Remote Sensing and Spatial Information Sciences 35 (Part B3), pp. 1091-1096.

Fischler, M. A., Bolles, R. C., 1981. Random sample consensus: A paradigm for model fitting with applications to image analysis and automated cartography. Communications of the ACM 24 (6), pp. 381-395.

Forkuo, E. K., King, B., 2004. Automatic fusion of photogrammetric imagery and laser scanner point clouds. International Archives of the Photogrammetry, Remote Sensing and Spatial Information Sciences 35 (Part B4), pp. 921-926.

Horn, B. K. P., Hilden, H. M., Negahdaripour, S., 1988. Closedform solution of absolute orientation using orthonormal matrices. Journal of the Optical Society of America A (5), pp. 1127-1135.

Kang, Z., Li, J., Zhang, L., Zhao, Q., Zlatanova, S., 2009. Automatic registration of terrestrial laser scanning point clouds using panoramic reflectance images. Sensors 9 (4), pp. 2621-2646.

Lepetit, V., Moreno-Noguer, F., Fua, P., 2009. EPnP: An accurate $\mathrm{O}(\mathrm{n})$ solution to the $\mathrm{PnP}$ problem. International Journal of Computer Vision 81 (2), pp. 155-166.

Lowe, D. G., 2004. Distinctive image features from scale-invariant keypoints. International Journal of Computer Vision 60 (2), pp. 91-110.

Magnusson, M., Lilienthal, A., Duckett, T., 2007. Scan registration for autonomous mining vehicles using 3D-NDT. Journal of Field Robotics 24 (10), pp. 803-827.

Moreno-Noguer, F., Lepetit, V., Fua, P., 2007. Accurate noniterative $\mathrm{O}(\mathrm{n})$ solution to the $\mathrm{PnP}$ problem. IEEE 11th International Conference on Computer Vision, pp. 1-8.

Nüchter, A., Gutev, S., Borrmann, D., Elseberg, J., 2011. Skylinebased registration of 3D laser scans. Geo-Spatial Information Science 14 (2), pp. 85-90.

Pathak, K., Birk, A., Vaskevicius, N., Poppinga, J., 2010a. Fast registration based on noisy planes with unknown correspondences for 3-D mapping. IEEE Transactions on Robotics 26 (3), pp. 424441.

Pathak, K., Borrmann, D., Elseberg, J., Vaskevicius, N., Birk, A., Nüchter, A., 2010b. Evaluation of the robustness of planar-patches based 3D-registration using marker-based ground-truth in an outdoor urban scenario. IEEE/RSJ International Conference on Intelligent Robots and Systems, pp. 5725-5730.

Rabbani, T., Dijkman, S., van den Heuvel, F., Vosselman, G., 2007. An integrated approach for modelling and global registration of point clouds. ISPRS Journal of Photogrammetry and Remote Sensing 61 (6), pp. 355-370.

Rusinkiewicz, S., Levoy, M., 2001. Efficient variants of the ICP algorithm. Proceedings of the Third International Conference on 3 D Digital Imaging and Modeling, pp. 145-152.

Stamos, I., Leordeanu, M., 2003. Automated feature-based range registration of urban scenes of large scale. IEEE Computer Society Conference on Computer Vision and Pattern Recognition, Vol. II, pp. 555-561.

Steder, B., Grisetti, G., Burgard, W., 2010. Robust place recognition for $3 \mathrm{D}$ range data based on point features. IEEE International Conference on Robotics and Automation, pp. 14001405.

Wang, Z., Brenner, C., 2008. Point based registration of terrestrial laser data using intensity and geometry features. International Archives of Photogrammetry, Remote Sensing and Spatial Information Sciences 37 (Part B5), pp. 583-589.

Weinmann, Ma., Weinmann, Mi., Hinz, S., Jutzi, B., 2011. Fast and automatic image-based registration of TLS data. ISPRS Journal of Photogrammetry and Remote Sensing. 